\title{
Impact of Concomitantly Administered Curcumin on Pharmacokinetics of Daclatasvir in Mice Under the Frame of Herb-Drug Interaction
}

\author{
Asmita Magotra, Pankul Kotwal, Shipra Bhatt, Ashish Dogra, Gurdarshan Singh, Utpal Nandi ${ }^{*}$ \\ PK-PD, Toxicology and Formulation Division, CSIR-Indian Institute of Integrative Medicine, Jammu, Jammu and Kashmir, INDIA.
}

\begin{abstract}
Objective: Daclatasvir is a new orally acting antiviral drug for the treatment of hepatitis $C$ virus (HCV). It is substrate of both P-glycoprotein (P-gp) and CYP3A4 which are involved in the major pharmacokinetic interaction between drug-drug or drug-food. Curcumin, a natural polyphenol extracted from Curcuma longa is used regularly and widely as food additive or dietary supplement. We surmised that curcumin may interfere with the pharmacokinetics of daclatasvir as curcumin is known to have potential to hinder the drug transporters and hepatic drug metabolising enzymes. Hence, investigations were taken up to assess for such possible interactions. Materials and Methods: Pharmacokinetics of daclatasvir after oral administration as alone and in combination with curcumin $(2.5$ or 5 or $10 \mathrm{mg} / \mathrm{kg}$ ) were investigated in Balb/C mice following determination of daclatasvir plasma concentration by using a sensitive LC-MS/MS method. Results: Area under the plasma concentration-time curve for daclatasvir was decreased at lowest dose and increased subsequently at higher doses upon concomitant administration of curcumin with daclatasvir in comparison to daclatasvir alone. Maximum plasma concentration of daclatasvir was enhanced only at highest dose. Overall effects of curcumin on daclatasvir pharmacokinetics were not statistically significant at all dose levels except significant increase in mean residence time. Conclusion: Intensity of curcumin effect on the daclatasvir pharmacokinetics at the experimental dose level in the mice model suggests that dose adjustment is unlikely to be required. Further experimentations are needed to confirm its clinical significance.
\end{abstract}

Key words: Daclatasvir, Curcumin, Pharmacokinetic interaction, Herb-drug interaction, Food-drug interaction.

\section{INTRODUCTION}

Daclatasvir is a new direct-acting antiviral agent for the treatment of chronic Hepatitis C Virus (HCV) infections. ${ }^{1}$ Daclatasvir $(60$ $\mathrm{mg}$ ) in combination with sofosbuvir (400 $\mathrm{mg}$ ) is recommended as first-line therapy in patients with genotype 3 without cirrhosis as per the recent report of American Association for the Study of Liver Diseases. ${ }^{2}$ It is given orally in its hydrochloride salt form and available in the market under the brand name of 'DAKLINZA'. ${ }^{3}$ On the other hand, curcumin is described as 'wonder drug in waiting' because of its multiple pharmacological activities with acceptable safety profile. This natural polyphenol is used regularly and widely as spice or food additive or dietary supplement namely turmeric. ${ }^{4}$ Curcumin have potential to cause inhibition of both P-glycoprotein (P-gp) and CYP isoenzymes. Moreover, curcumin can be taken by the people inadvertently and frequently with prescription and nonprescription allopathic drugs without the knowledge of their health care providers. ${ }^{5,6}$ This may lead to drug-drug or drugfood interactions when administered with a substrate of P-gp and CYP3A4 like daclatasvir. ${ }^{2}$ Therefore, it is pertinent to investigate the daclatasvir plasma levels upon concomitant administration of curcumin
Submission Date: 06-03-2018; Revision Date: 17-05-2018; Accepted Date: 22-05-2018

DOI: 10.5530/ijper.52.4s.70 Correspondence: Utpal Nandi, PK-PD, Toxicology and Formulation Division, CSIRIndian Institute of Integrative Medicine, Jammu, Jammu \& Kashmir 180 001, INDIA. Phone: +91-0191-2569000 Fax: +91- 0191-2569333 E-mail: unandi@iiim.ac.in

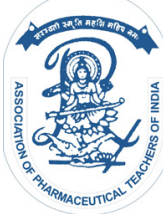

www.ijper.org 
with daclatasvir in animal model as it can lead to serious consequence like treatment failure or beneficial consequences like daclatasvir dose reduction and safe administration with curcumin. Results of these preclinical investigations ultimately helpful before going into costly and perilous clinical investigation. ${ }^{5-7}$

There is no report on pharmacokinetic interaction of such a new drug like daclatasvir with curcumin in mice model till date. Hence, the objective of our present study to explore the effect of curcumin (2.5 to $10 \mathrm{mg} / \mathrm{kg}$ ) on oral pharmacokinetics of daclatasvir dihydrochloride $(14 \mathrm{mg} / \mathrm{kg})$ in mice.

\section{MATERIALS AND METHODS Materials}

Curcumin (purity $\geq 99.0 \%$ ) was purchased from Loba Chemie. Ammonium formate, solutol HS15, dimethyl sulfoxide was purchased from Sigma-Aldrich. All other chemicals used were of research grade. Ultrapure water (Millipore) was used throughout the experimentations.

\section{Ethical prerequisites}

All animal experimentations were performed with 'Committee for the Purpose of Control and Supervision of Experiments on Animals' (CPCSEA) guidelines (Govt. of India, New Delhi India) after obtaining necessary approval from Institutional Animal Ethics Committee of CSIR-Indian Institute of Integrative Medicine (IAEC approval no: 70/104/2/17).

\section{Animal's husbandry and maintenance}

Pharmacokinetic studies were carried out using healthy adult male Balb/C mice, which were housed in PP cages under standard laboratory conditions like temperature of $25 \pm 2^{\circ} \mathrm{C}$, relative humidity of $50 \pm 20 \%$, light cycle of $12 \mathrm{~h} / 12 \mathrm{~h}$, fed with pellet diet and water ad libitum.

\section{Dose selection and preparation}

Dose of daclatasvir dihydrochloride $(14 \mathrm{mg} / \mathrm{kg})$ was selected for mice on the basis of its human daily dose of $66 \mathrm{mg}$ that is equivalent to $60 \mathrm{mg}$ of daclatasvir. Whereas, dose of curcumin $(2.5 / 5 / 10 \mathrm{mg} / \mathrm{kg})$ was on the basis of bioenhancer mechanism which function at low doses. ${ }^{8}$ Individual dose was prepared as solution form using 10\% DMSO, 20\% solutol HS15 and water (q.s) for oral administration of curcumin and daclatasvir dihydrochloride.

\section{Experimental design}

Pharmacokinetic studies were performed following single dose oral administration of daclatasvir dihydrochloride $(14 \mathrm{mg} / \mathrm{kg})$ as alone and in combination with curcumin at three dose level viz. $2.5 \mathrm{mg} / \mathrm{kg}, 5 \mathrm{mg} / \mathrm{kg}$ and $10 \mathrm{mg} / \mathrm{kg}$. Each study was done by using total twenty animals, divided into four subgroups for sparse sampling $(\mathrm{n}=5)$ i.e. two blood samples to be withdrawn at different time points from one subgroup. Animals were fasted for $10 \mathrm{~h}$ prior to experimentation with free access to water. Curcumin was administered $0.5 \mathrm{~h}$ before daclatasvir dosing. Blood samples were collected with anticoagulant at $0 \mathrm{~h}, 0.25 \mathrm{~h}, 0.5 \mathrm{~h}, 1 \mathrm{~h}, 2 \mathrm{~h}, 4 \mathrm{~h}$, $8 \mathrm{~h}$ and $12 \mathrm{~h}$ after oral administration of daclatasvir dihydrochloride. Plasma was separated using centrifugation and stored at $-20^{\circ} \mathrm{C}$ until analysis.

\section{Plasma sample analysis by LC-MS/MS}

Stock solution $(1 \mathrm{mg} / \mathrm{mL})$ was prepared in methanol for daclatasvir and sofosbuvir (Internal Standard) and diluted further with acetonitrile to prepare standard solutions. A ten point calibration curve of analyte was prepared by spiking serially diluted working solution into blank plasma to obtain final concentrations in the range of 3.9, 7.8, 15.6, 31.25, 62.5, 125, 250, 500, 1000 and $2000 \mathrm{ng} / \mathrm{mL}$ for daclatasvir. Simple protein precipitation procedure was employed to recover analytes from mice plasma. Plasma sample $(50 \mu \mathrm{L})$ was processed by adding IS $(10 \mu \mathrm{L}$ of $20 \mu \mathrm{g} / \mathrm{mL})$ followed by vortex mixing for $2 \mathrm{~min}$. Then, $190 \mu \mathrm{L}$ of acetonitrile was added and centrifugation was done at $14000 \mathrm{rpm}$ for $10 \mathrm{~min}$. Supernatant layer was separated and injected into the LC-MS/MS (Shimadzu, Kyoto, Japan) system for quantitation of daclatasvir in mice plasma. Chromatographic separation was achieved in Hiber ${ }^{\circledR}$ HR RP-18 (50 x 2.1 $\mathrm{mm}, 3 \mu \mathrm{m}$ ) column (Merck, India) using isocratic mobile phase composition of $10 \mathrm{mM}$ ammonium formate at $\mathrm{pH}$ adjusted to 3.5 and acetonitrile $(50: 50, \% \mathrm{v} / \mathrm{v})$. Flow rate was kept at $0.3 \mathrm{~mL} / \mathrm{min}$ and column oven temperature was maintained at $30^{\circ} \mathrm{C}$. Triple-quadrupole tandem mass spectrometer was operating in positive ion mode and quantification was done in multiple reaction monitoring mode using precursor/product ion transition at $\mathrm{m} / \mathrm{z}$ 739.55/565.35 and 530.30/243.10 for daclatasvir and sofosbuvir, respectively. Data were acquired and processed using Lab Solutions software. Quantitation of daclatasvir in mice plasma was performed by following LC-MS/MS methods. ${ }^{9}$

\section{Pharmacokinetic data analysis}

Plasma concentration data of daclatasvir with respect to time was processed by non-compartmental method using PK solution software (Summit Research Services, USA). Based on the dose of daclatasvir equivalent to $14 \mathrm{mg} / \mathrm{kg}$ daclatasvir dihydrochloride, different 
pharmacokinetic parameters were calculated to compare the results of different treatment groups.

\section{Statistical evaluation}

Statistical analysis was performed using Student's t-test (QuickCals, Prism online version) by comparing data (mean \pm SEM) of combination treatment of curcumin and daclatasvir group with daclatasvir alone treated group. Data considered to be significant if $\mathrm{p}<0.05$.

\section{RESULTS}

Pharmacokinetic studies of daclatasvir dihydrochloride $(14 \mathrm{mg} / \mathrm{kg})$ as alone and concomitantly administered with curcumin at 2.5 or 5 or $10 \mathrm{mg} / \mathrm{kg}$ in Balb/C mice were performed. Daclatasvir concentration in plasma was quantified by a sensitive LC-MS/MS method and mean plasma concentrations of daclatasvir versus time profiles are represented in Figure 1. Main pharmacokinetic parameters were calculated based on daclatasvir dose equivalent to $14 \mathrm{mg} / \mathrm{kg}$ of daclatasvir dihydrochloride and summarised in Table 1 . Results showed that time to reach maximum plasma concentration $\left(\mathrm{T}_{\max }\right)$ was 2-4 h with maximum plasma concentration $\left(\mathrm{C}_{\max }\right)$ of $1360 \pm 68 \mathrm{ng} / \mathrm{mL}$ upon individual administration of daclatasvir. Area under the curve for plasma concentration of daclatasvir from zero to the last measurable plasma sample time and to infinity $\left(\mathrm{AUC}_{0-\mathrm{t}}\right.$ and $\left.\mathrm{AUC}_{0-\infty}\right)$ were $7883 \pm 476$ and $8210 \pm 521 \mathrm{ng} \cdot \mathrm{h} / \mathrm{mL}$, respectively when daclatasvir was administered as alone. Volume of distribution $\left(\mathrm{V}_{\mathrm{d}}\right)$ and clearance $(\mathrm{Cl})$ for daclatasvir upon individual administration were $4.8 \pm 1.6 \mathrm{~L} / \mathrm{kg}$ and $1.6 \pm 0.1 \mathrm{~L} / \mathrm{h} / \mathrm{kg}$, respectively. $\mathrm{C}_{\max }(1198 \pm 27 \mathrm{ng} / \mathrm{mL})$ and $\mathrm{AUC}_{0-\infty}(7706 \pm 613 \mathrm{ng} \cdot \mathrm{h} / \mathrm{mL})$ of daclatasvir were changed upon concomitant administration of curcumin at $2.5 \mathrm{mg} / \mathrm{kg}$ but it is not statistically significant. When increased the dose of curcumin to $5 \mathrm{mg} / \mathrm{kg}$, daclatasvir pharmacokinetic parameters were similar to daclatasvir

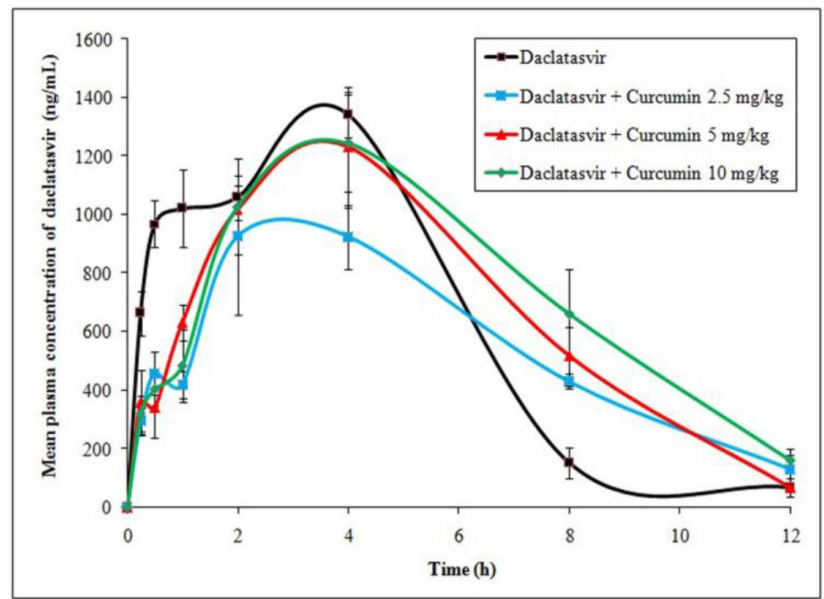

Figure 1: Plasma concentration (mean $\pm \mathrm{SEM}$ ) versus time profiles of daclatasvir as alone and concomitantly administered with curcumin through oral route in Balb/C mice.

alone group except decreased in $\mathrm{C}_{\max }(1286 \pm 186 \mathrm{ng} / \mathrm{mL})$. These changes in plasma level have no statistical significance. $\mathrm{AUC}_{0-\infty}(9377 \pm 698 \mathrm{ng} \cdot \mathrm{h} / \mathrm{mL})$ of daclatasvir was improved upon further augmented dose of $10 \mathrm{mg} / \mathrm{kg}$ but none of the parameters were statistically significant as compared to daclatasvir alone group except mean residence time (MRT). MRT of daclatasvir was enhanced significantly with concomitant administration of curcumin.

\section{DISCUSSION}

Daclatasvir is also a substrate of P-gp and primarily metabolised through CYP3A enzyme. ${ }^{1-3}$ We investigated the consequences of concurrent administration of daclatasvir and curcumin, an inhibitor of P-gp, organic anion transporting polypeptide (OATP) 1B1 and 1B3, and breast cancer resistance protein (BCRP). ${ }^{4}$ Plasma level of daclatasvir was increased maximum up to $3 \%$ and $14 \%$ upon curcumin treatment at $5 \mathrm{mg} / \mathrm{kg}$ and $10 \mathrm{mg} / \mathrm{kg}$, respectively. Sometimes, this type of positive pharmacokinetic interactions beyond certain degree are

\begin{tabular}{|c|c|c|c|c|}
\hline $\begin{array}{l}\text { Pharmacokinetic } \\
\text { parameter }\end{array}$ & Daclatasvir & $\begin{array}{c}\text { Daclatasvir }+ \\
\text { Curcumin } \\
(2.5 \mathrm{mg} / \mathrm{kg})\end{array}$ & $\begin{array}{c}\text { Daclatasvir + } \\
\text { Curcumin } \\
\text { (5 mg/kg) }\end{array}$ & $\begin{array}{c}\text { Daclatasvir }+ \\
\text { Curcumin } \\
\text { (10 mg/kg) }\end{array}$ \\
\hline $\mathrm{C}_{\max }(\mathrm{ng} / \mathrm{mL})$ & $1360 \pm 68$ & $1198 \pm 27$ & $1286 \pm 186$ & $1409 \pm 113$ \\
\hline $\mathrm{T}_{\max }(\mathrm{h})$ & $3.6 \pm 0.4$ & $2.8 \pm 0.5$ & $3.2 \pm 0.5$ & $4.4 \pm 1.0$ \\
\hline $\mathrm{AUC}_{0-\mathrm{t}}(\mathrm{ng} \cdot \mathrm{h} / \mathrm{mL})$ & $7883 \pm 476$ & $6904 \pm 233$ & $8108 \pm 1138$ & $8823 \pm 709$ \\
\hline $\mathrm{AUC}_{0-\infty}(\mathrm{ng} \cdot \mathrm{h} / \mathrm{mL})$ & $8210 \pm 521$ & $7706 \pm 613$ & $8256 \pm 1128$ & $9377 \pm 698$ \\
\hline $\mathrm{V}_{\mathrm{d}}(\mathrm{L} / \mathrm{kg})$ & $4.8 \pm 1.6$ & $6.3 \pm 1.7$ & $3.9 \pm 1.1$ & $4.6 \pm 1.1$ \\
\hline $\mathrm{Cl}(\mathrm{L} / \mathrm{h} / \mathrm{kg})$ & $1.6 \pm 0.1$ & $1.7 \pm 0.1$ & $1.7 \pm 0.2$ & $1.6 \pm 0.2$ \\
\hline MRT(h) & $3.7 \pm 0.3$ & $5.9 \pm 0.9$ & $4.6 \pm 0.1$ & $5.5 \pm 0.4$ \\
\hline
\end{tabular}


deliberately used to improve the exposure of drugs that also eventually beneficial for poor bioavailable drugs or drugs having dose dependent side effects to reduce dose. $^{8}$ Common mechanisms of curcumin for pharmacokinetic interactions as evidenced from literature are modulated through down regulation of CYP450, followed by P-gp and a few downstream pathways involving OATP, glutathione-S-transferase (GST) and uridine dinucleotide phosphate glucuronosyltransferases (UDPG). Based on in-vitro studies reported data, curcumin could inhibit $\mathrm{P}$-gp in dose dependent manner at micromolar concentration range and inhibit CYP3A4 competitively with low potency of more than $10 \mu \mathrm{M} .{ }^{4}$ On the other hand, curcumin at lowest experimental dose $(2.5 \mathrm{mg} / \mathrm{kg}$ ) might have poor efficacy to up regulate absorption and down regulate metabolism of daclatasvir. This fall in plasma concentration level of daclatasvir upon coadministration of curcumin can lead to treatment failure due to negative pharmacokinetic interaction. Severity of disease conditions have many a times been aggravated and developed resistance of chemotherapeutic agents. This may be due to a large portion of curcumin entering the intestinal epithelium is extensively metabolized intracellularly and therefore, P-gp binding is reduced. ${ }^{10}$ On the contrary, quite a few recent investigations appeared to indicate unexpectedly the opposite results related to complex $\mathrm{ABC}$ transporter systems. ${ }^{11,12}$ Overall effect may be correlated to low level of P-gp by curcumin might be compensated by over expression of other transporters having an affinity for daclatasvir. ${ }^{10}$ Results obtained in the present studies are encouraging as net effects of daclatasvir upon concurrent administration with curcumin were not affected to a greater extent. Nevertheless, some further studies should be carried out to ascertain the experimental outcomes.

\section{CONCLUSION}

Pharmacokinetic studies of daclatasvir after oral administration in presence or absence of curcumin in Balb/C mice revealed that there is no significant effect of curcumin on daclatasvir pharmacokinetics except increase in mean residence time. This effect of curcumin on daclatasvir pharmacokinetics has considerable impact on limitation of the concomitant use of curcumin or requirement of dose adjustment for daclatasvir. Additional preclinical investigations are needed to go into the fathom of the idea.

\section{ACKNOWLEDGEMENT}

Authors are grateful to Director (CSIR-IIIM) to carry out this research work which was supported by CSIR, New Delhi, India (MLP6006). AM, PK, AD and SB are thankful to DST, UGC and CSIR for providing research fellowships. IIIM Publication No: IIIM/2169/2017

\section{CONFLICT OF INTEREST}

The authors declare no confict of interest.

\section{ABBREVIATIONS}

HCV: Hepatitis C virus; P-gp: P-glycoprotein; LC-MS / MS: Liquid chromatography tandem mass spectrometry; $\mathbf{C}_{\max }$ : Maximum plasma concentration; $\mathbf{T}_{\max }$ : Time to reach maximum plasma concentration; MRT: Mean residence time; $\mathbf{A U C}_{0-\mathrm{t}}$ : Area under the curve for plasma concentration from zero to the last measurable plasma sample time; $\mathbf{A U C}_{\mathbf{0}-\infty}$ : Area under the curve for plasma concentration from zero to time infinity; $\mathbf{V}_{\mathrm{d}}$ : Volume of distribution; Cl, Clearance; OATP: Organic anion transporting polypeptide; BCRP: Breast cancer resistance protein; GST: Glutathione-S-transferase; UDPG: Uridine dinucleotide phosphate glucuronosyltransferases.

\section{REFERENCES}

1. Soriano V, Labarga P, Fernandez-Montero JV, Mendoza CD, BenitezGutierrez L, Pena JM, et al. Drug interactions in HIV-infected patients treated for hepatitis C. Expert Opin Drug Metab Toxicol. 2017;13(8):807-16.

2. Manolakopoulos S, Zacharakis G, Zissis M., Giannakopoulos V. Safety and efficacy of daclatasvir in the management of patients with chronic hepatitis $C$. Ann Gastroenterol. 2016;29(3):282-96.

3. Bifano M, Adamczyk R, Hwang C, Kandoussi H, Marion A, Bertz RJ. An open-label investigation into drug-drug interactions between multiple doses of daclatasvir and single-dose cyclosporine or tacrolimus in healthy subjects. Clin Drug Investig. 2015;35(5):281-9.

4. Bahramsoltani R, Rahimi R, Farzaei MH. Pharmacokinetic interactions of curcuminoids with conventional drugs: A review. J Ethnopharmacol 2017;209:1-12

5. Tarirai C, Viljoen AM, Hamman JH. Herb-drug pharmacokinetic interactions reviewed. Expert Opin Drug Metab Toxicol. 2010;6(12):1515-38.

6. Ekor M. The growing use of herbal medicines: Issues relating to adverse reactions and challenges in monitoring safety. Front Pharmacol. 2013;4:1-10.

7. Srinivas NR. Recent trends in preclinical drug-drug interaction studies of flavonoids-Review of case studies, issues and perspectives. Phytother Res. 2015;29(11):1679-91.

8. Atal N, Bedi KL. Bioenhancers: Revolutionary concept to market. J Ayurveda Integr Med 2010;1(2):96-9.

9. Rezk MR, Bendas ER, Basalious EB, Karim IA. Development and validation of sensitive and rapid UPLC-MS/MS method for quantitative determination of daclatasvir in human plasma: Application to a bioequivalence study. J Pharm Biomed Anal. 2016;128:61-6. 
11. Gutmann H, Fricker G, Drewe J. Interactions of HIV protease inhibitors with ATP-dependent drug export proteins. Mol Pharmacol. 1999;56(2):383-9.

12. Cascorbi I. Role of pharmocogenetics of ATP-binding cassette transporters in the pharmacokinetics of drugs. Pharmacol Ther. 2006;112(2):457-73.

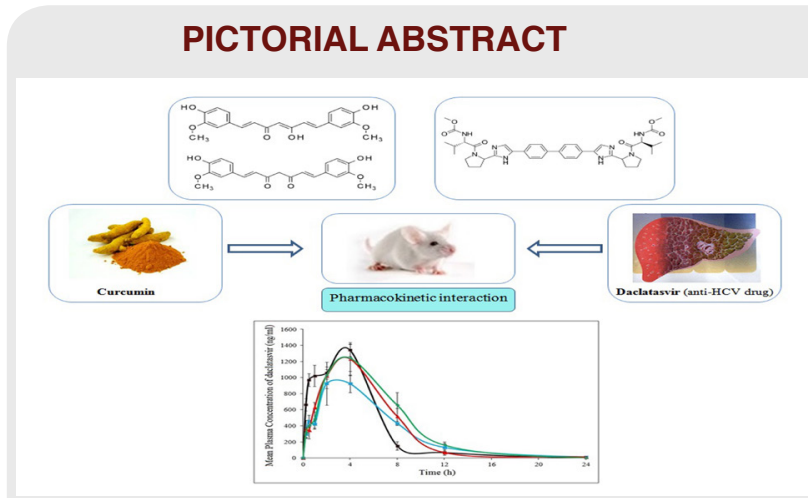

\section{About Authors}
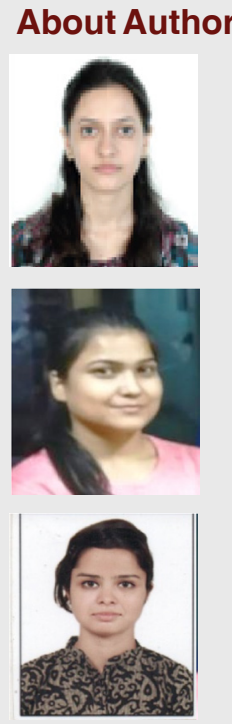

Asmita Magotra: She is pursuing her Ph. D. degree in the field of pharmacokinetics and pharmacodynamics from CSIR-Indian Institute of Integrative Medicine, Jammu.

- Concomitant administration daclatasvir and curcumin have potential for drug-food or drug-drug interaction through modulation of P-glycoprotein and CYP3A4 enzymes. Curcumin could not altered daclatasvir pharmacokinetics significantly except increase in mean residence time upon concomitant single dose oral administration. Pharmacokinetics of daclatasvir should be explored upon pretreatment for several days with curcumin in preclinical model and then clinical significance needs to be investigated.

Pankul Kotwal: She is working as Junior Research Fellow (UGC) to pursue her Ph. D. in the field of pharmacokinetics from CSIR-Indian Institute of Integrative Medicine, Jammu.

Shipra Bhatt: She is working as Junior Research Fellow (CSIR) to pursue her Ph. D. degree in the field of drug metabolism from CSIR-Indian Institute of Integrative Medicine, Jammu.
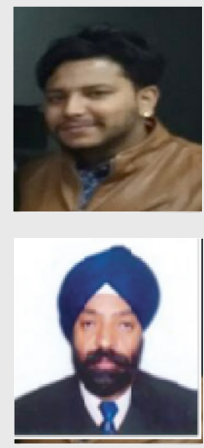

Ashish Dogra: He is working as Junior Research Fellow (UGC) to pursue his Ph. D. degree in the field of pharmacokinetics and toxicity from CSIR-Indian Institute of Integrative Medicine, Jammu.

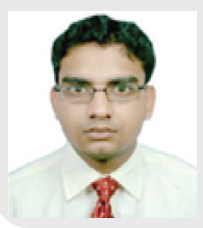

Gurdarshan Singh: $\mathrm{He}$ is presently working as Principal Scientist in CSIR-Indian Institute of Integrative Medicine, Jammu in the area of Pharmacokinetics, Pharmacodynamics and Toxicology.

Cite this article: Magotra A, Kotwal P, Bhatt S, Dogra A, Singh G, Nandi U. Impact of Concomitantly Administered Curcumin on Pharmacokinetics of Daclatasvir in Mice Under the Frame of Herb-Drug Interaction. Indian $\mathrm{J}$ of Pharmaceutical Education and Research. 2018;52(4S):S11-S15. 